\title{
Rethinking transport
}

\section{7-30 April 2020}

Proceedings of 8th Transport Research Arena TRA 2020, April 27-30, 2020, Helsinki, Finland

\section{Evaluation of current European open science initiatives in transport research}

\author{
Afroditi Anagnostopoulou ${ }^{\mathrm{a}}$, Attila Akac ${ }^{\mathrm{a} *}$, Maria Boile $^{\mathrm{b}}$, \\ ${ }^{a}$ Hellenic Institute of Transport, Centre for Research and Technology Hellas, G. Kassimati 1, Piraeus 18531, Greece \\ ${ }^{b}$ Hellenic Institute of Transport, Centre for Research and Technology Hellas, Aigialeias 52, Marousi 15125, Greece
}

\begin{abstract}
Latest technological developments have endorsed the creation of new collaborative tools and enabling transport research community information sharing. Following these technological directives, European Commission is continuously developing appropriate initiatives that aim at making science more efficient and reproducible to societal and economical expectations. This paper conducts an analysis and evaluation of exploited Open Science initiatives and open access platforms in Europe. The proposed analysis presents clusters of possible use areas with respect to different challenges that key actors in transport research may face. The focus is given on the evaluation of different combined factors which could be used as a qualitative measurement for existing Open Science tools. Subsequently, relevant stakeholders could acknowledge the existence of Open Science initiatives and the way to use them efficiently in order to enhance a collaborative, innovative and transparent research environment.
\end{abstract}

Keywords: open science; transport research; evaluation

\footnotetext{
* Corresponding author. Tel.: +302-110-130-489;

E-mail address: akac.attila@certh.gr
} 
Nomenclature

$\begin{array}{ll}\text { OS } & \text { Open Science } \\ \text { EOSC } & \text { European Open Science Cloud } \\ \text { EC } & \text { European Commission } \\ \text { EU } & \text { European Union } \\ \text { IETF } & \text { Internet Engineering Task Force } \\ \text { GIPO } & \text { Global Internet Policy Observatory } \\ \text { TRIMIS } & \text { Transport Research and Innovation Monitoring and Information System } \\ \text { CORDIS } & \text { Community Research and Development Information Service } \\ \text { TRL } & \text { Technology Readiness Level }\end{array}$

\section{Introduction}

Open Science (OS) is a modern movement that represents a new approach to practicing science, in a way that increases openness, integrity and reproducibility of research. It aims at giving everyone access in scientific process and results in a more transparent and accessible way at all levels. The rapid growth of digital technologies and new collaborative tools become enablers of OS, allowing speed up the process of adopting open habits and facilitating the sharing of large volumes of information, study materials and data, as it stated by FOSTER (2018). Europe has cultivated a culture to share research activities across national boundaries, which put it in a leading position in the world to promote and expedite the new OS way of working. In fact, nearly $10 \%$ of the budget allocated for the Horizon 2020 Work Programme 2018-2020 is channeled to direct or indirect support of OS and towards this direction, the EOSC (2018) has been developed to enable sharing and re-use of research data across disciplines and borders, taking into account relevant legal, security and privacy issues.

In the context of transport, the European Commission Transportation Research and Innovation policy intends to foster OS through user engagement enabling dynamic knowledge circulation, as mentioned by Tsakalidis et al. (2018). As such, an "Expert Group on Open Science - Transport Research Cloud" (2017) has been established to assess the need to create a transport research cloud. Moreover, two research projects (BE OPEN "European forum and oBsErvatory for OPEN science in transport" and OSCAR "Open ScienCe Aeronautic \& Air Transport Research") have been recently funded by the European Commission in an attempt to support shifting from the standard practices of publishing research results in scientific publications towards sharing and using all available knowledge at an earlier stage in the research process. Anagnostopoulou and Boile (2018) studied that research, technology and innovation actions in the transport sector face new challenges linked to the aspects of OS. Technical interoperability (C1), code of implementation such as membership and governance of new open observatories and forums $(C 2)$, common terminology to support data and information interoperability (C3), deployment of new skills and competencies for practicing Open Science $(C 4)$, as well as the adoption of collaborative ways of working (C5) are the main challenges of building OS platforms in transport research.

This paper conducts an analysis and evaluation of exploited Open Science initiatives and open access platforms in Europe. The proposed analysis presents clusters of possible use areas with respect to different challenges that key actors in transport research may face. The focus is given on the evaluation of different combined factors which could be used as a qualitative measurement for existing Open Science tools. Subsequently, relevant stakeholders could acknowledge the existence of Open Science initiatives and the way to use them efficiently in order to enhance a collaborative, innovative and transparent research environment. The remainder of the paper is organized as follows: Section 2 describes the benefits offered to scientists and citizens, the limitations that users face and the services provided by each OS platform. Then, Section 3 analyzes how OS initiatives could offer services to the proposed clusters of competence areas and summarizes the overall coverage of the main challenges by the existing OS platforms in transport research. Finally, in Section 4 conclusions are drawn and pointers for future research are provided. 


\section{Evaluation of Open Science initiatives}

In recent years, the need for open data, unified standards and flexible infrastructures in the research area continues to grow at faster rates and thus, a number of initiatives have developed technical background, policy information and shared knowledge in different domains of transport sector. OS platforms contain different classes of services, which are based on databases or inventories, projects and publications. Transport stakeholders and other relevant key actors (from transport industry and research community, European Commission and society in general) are major contributors in EC's actions that strive towards an open access and integrated research sharing ground. The relevant stakeholders, consequently users of OS services, have a two-fold role during their contribution in improvement of OS services. On one hand, they have strong position and major influence on the enhancement of current or future initiatives according to their competence area. On the other hand, they utilize published knowledge and tools to solve various use case scenarios (according to their field of interest or competence).

Therefore, the current OS initiatives supported by EC and their embedded services in transport research area are summarized as follows:

(i) the European Open Science Cloud (EOSC) - Transport Research Cloud is an initiative which kicked-off in 2016 as a part of European Cloud Initiative to build a competitive data and knowledge economy in Europe. The EOSC aims to rely on existing and emerging data infrastructures and cloud-based services to create an easily accessible entry point for everyone interested to store, manage, analyze and re-use existing research data. Currently, EC is providing financial support to implement the EOSC by various ongoing and future projects under the EU Framework Programme for Research and Innovation (Horizon 2020) that will contribute to the development of the EOSC by 2021 (EOSC, 2018).

(ii) OpenAIRE-Advance is closely linked to the EOSC initiative and partially follows the EOSC vision. OpenAIRE is aiming at providing unlimited, unrestricted and open access to research outputs for every citizen, educator and industry representative. Through monitoring and support on national policies and protocols implementation, training support on national open access desks and open value added services, OpenAIRE has structured separate dashboards specified for the exploitation, publication and development of available EU research data (OpenAIRE portal services, 2016).

(iii) the Internet Engineering Task Force (IETF) is a large open international community of network designers, operators, vendors, and researchers concerned with the evolution of the Internet architecture and the smooth operation of the Internet. Partners of this community pursue the promotion of open access and voluntary work amongst different technical competence areas of research society through specific standards and protocols. The current Task Force specializes mostly on data management, data security and interoperability issues which are directly affecting numerous of research and industrial sectors globally (IETF, 2014).

(iv) the Research Data Alliance (RDA) was launched as a community-driven initiative in 2013 by the EC, the United States Government's National Science Foundation and National Institute of Standards and Technology, and the Australian Government's Department of Innovation with the goal of building the social and technical infrastructure to enable open sharing and re-use of data. RDA provides a neutral c-working space, where its members can come together through focused global Working and Interest Groups to create a cohesive data community for all contributors globally (Research Data Alliance, 2019).

(v) the Fostering the practical implementation of Open Science in Horizon 2020 and beyond - (FOSTER) project which is an e-learning portal that provides training resources related to OS and develops strategies and skills for the proper OS practices implementation. Many different users - from early-career researchers, to data managers, librarians, research administrators, and graduate schools - can benefit from the portal and through a developed training assessment plan to enhance OS activities in different research sectors. The content of this platform is based on different elements of OS like open access, open data, OS policies and tools and OS evaluation processes. Respectively, FOSTER provides supplementary research data management, data mining and regulatory content for the EU research community in order to comply on the specified taxonomies (FOSTER, 2018).

(vi) In the field of Open Science in transport research, an important coordinated approach of the European Commission is the launch of the Transport Research and Innovation Monitoring and Information System 
(TRIMIS) which aims at becoming the EC's tool for mapping technology trends and research and innovation capacities in the transport field.. TRIMIS provide open-access information on seven thematic transport research areas, such as electrified and automated transport, low-emission alternative transport, smart mobility and infrastructure and network and traffic management. It is designed as an open-access information and knowledge management system that also provides different roadmaps of innovation and new technology in transport (TRIMIS, 2019).

(vii) Additionally, the Community Research and Development Information Service (CORDIS) is the European Commission's primary source of results from the projects funded by the EU's framework programmes for research and innovation (FP1 to Horizon 2020) since 1990. The CORDIS aims to make research results accessible to researchers, within the context of fostering Open Science. CORDIS has a rich and structured public repository with all information on projects held by the European Commission, such as project factsheets, participants, reports, deliverables and links to open-access publications, while also produces its own range of publications and articles, in order to facilitate the user to find relevant results in each domain (CORDIS, 2019)..

In the context of Open Access in transport research, publishing companies realized that opening up European research could stimulate greater movement of knowledge and promote excellence improving cooperation between research community and industry (Anagnostopoulou and Boile, 2018). The open access platforms that could support the European OS actions are (i) Springer, (ii) Elsevier, (iii) Hindawi, in collaboration with John Wiley \& Sons Inc., (iv) Taylor \& Francis, (v) Scipedia, (vi) the Directory of Open Access Journals (DOAJ), (vii) InderScience Publishers, and (viii) University domain publications (i.e. EJTR). Open access platforms and EC services intend to give a transparent and accessible research industry to everyone, although create some challenges that have to be faced in the future. These limitations and the relevant cluster of scenarios that these platforms tend to encounter will be analyzed in the following section.

\section{Analysis of Open Science initiatives coverage areas}

The latest available transport research studies show that OS related initiatives over the last 40 years are limited (less than 100 relevant sources coming from EU research community) showing a preference towards use cases applied in multimodal transportation sector (BE OPEN (b), 2019). These sources validated the existence of the above-mentioned OS initiatives as the most commonly used in EU research transport sector. Nevertheless, stakeholder needs and objectives are constantly changing throughout time and pushes researchers to periodically evaluate the current state of transport domain. For this reason, a targeted desk research was conducted in the context of the BE OPEN project (BE OPEN (b), 2019) with the participation of 14 transport stakeholders in order to map the current penetration of OS in transport research sector. The aforementioned deliverable of the BE OPEN project pointed out the further need in EU coordinated actions to create the appropriate policy and regulatory mechanism to promote OS usage and ensure their proper implementation (GDPR, national legal issues). Accordingly, data handling services and operational level (TRL 7) applications should be promoted equally in a structured open access system.

Dealing with the different limitations and challenges of OS (as they mentioned in Section 1), exploiting the results of the BE OPEN project (BE OPEN Project (b), BE OPEN (c), 2019) and based on the opportunities that OS initiatives offer (as they mentioned in Section 2), stakeholders could use OS in an efficient and effective way in order to solve their real-life problems. As such, three main clusters of use areas (Fig. 1) (i.e. (i) Services \& applications, (ii) Infrastructure and (iii) Policies \& regulations) are proposed to present how OS tools cover the different challenges and in addition, to provide a qualitative measurement for existing OS initiatives. These clusters have been assessed during a set of targeted actions with the help from different main actors' transport stakeholders (European and national research entities) in order to specify use area categories in a simple way. Specifically, the services \& applications refer to tools and components that offer assistance or carry out a set of procedures; infrastructure refers to technical facilities and systems servicing users and policies \& regulations refer to specifications, policies, standards or laws. 
Fig. 1 Use Areas

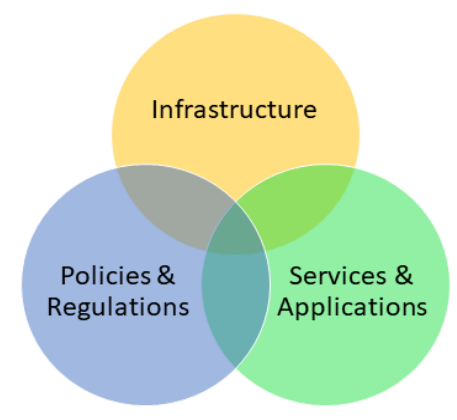

The proposed analysis could support a potential user or stakeholder to understand which main challenges are covered by the existing OS initiatives and in which use area the different OS tools could be used efficiently in order to support stakeholders' activities. According to the existing OS initiatives in European area, an overall table (see Table 1) specifies the relation of each initiative with the associated pair of factors examined.

Table 1. Analysis of European Open Science initiatives.

\begin{tabular}{|c|c|c|}
\hline OS initiatives & Tackled main challenges & Cluster use areas \\
\hline EOSC & $\mathrm{C} 1, \mathrm{C} 2, \mathrm{C} 3, \mathrm{C} 4, \mathrm{C} 5$ & $\begin{array}{l}\text { - Services \& applications } \\
\text { - Infrastructure } \\
\text { - Policies \& regulations }\end{array}$ \\
\hline OpenAIRE & $\mathrm{C} 1, \mathrm{C} 2, \mathrm{C} 3, \mathrm{C} 4, \mathrm{C} 5$ & $\begin{array}{l}\text { - Services \& applications } \\
\text { - Infrastructure } \\
\text { - Policies \& regulations }\end{array}$ \\
\hline IETF & $\mathrm{C} 1, \mathrm{C} 2, \mathrm{C} 5$ & $\begin{array}{l}\text { - Infrastructure } \\
\text { - Policies \& regulations }\end{array}$ \\
\hline RDA & $\mathrm{C} 1, \mathrm{C} 2, \mathrm{C} 3, \mathrm{C} 5$ & $\begin{array}{l}\text { - Services \& applications } \\
\text { - Policies \& regulations }\end{array}$ \\
\hline TRIMIS & $\mathrm{C} 1, \mathrm{C} 2, \mathrm{C} 5$ & $\begin{array}{l}\text { - Services \& applications } \\
\text { - Infrastructure } \\
\text { - Policies \& regulations }\end{array}$ \\
\hline CORDIS & $\mathrm{C} 1, \mathrm{C} 2, \mathrm{C} 5$ & $\begin{array}{l}\text { - Services \& applications } \\
\text { - Infrastructure } \\
\text { - Policies \& regulations }\end{array}$ \\
\hline FOSTER & $\mathrm{C} 1, \mathrm{C} 4, \mathrm{C} 5$ & $\begin{array}{l}\text { - Services \& applications } \\
\text { - Infrastructure }\end{array}$ \\
\hline Open Access platforms & $\mathrm{C} 1, \mathrm{C} 4$ & $\begin{array}{l}\text { - Infrastructure } \\
\text { - Policies \& regulations }\end{array}$ \\
\hline
\end{tabular}

\section{Engangement of transport stakeholders in OS}

In an attempt to enhance openness, transparency, fairness and reproducibility of science, the proposed cluster use areas will establish the ground rules for the transport research communities, ultimately establishing a community of transport research organizations, willing to work on the basis of a commonly agreed "Open Science Code of 
Conduct". As such, within the implementation of the BE OPEN project both key transport stakeholders and OS related communities i.e. ECTRI (multimodal transport), FEHRL (road transport), WEGEMT (maritime transport), EURNEX (rail transport), DLR (air transport), EATEO (air transport), UITP (multimodal transport), HUMANIST (multimodal transport) and OpenAIRE (OS) are collaborating in a two-fold action plan: to engage them in a participatory approach fostering a dialogue on Open Science (what exists, what should be done, how it should be done) among relevant stakeholders in Europe and around the world, and develop a detailed roadmap for the implementation of sustainable open science modules which include key practices, infrastructures, policies and business models, all taking into account the specificities of the transport research domain, and the use and integration of existing-infrastructures and the emerging EOSC initiative.

Fig. 2 Awareness of Transport Stakeholders in OS

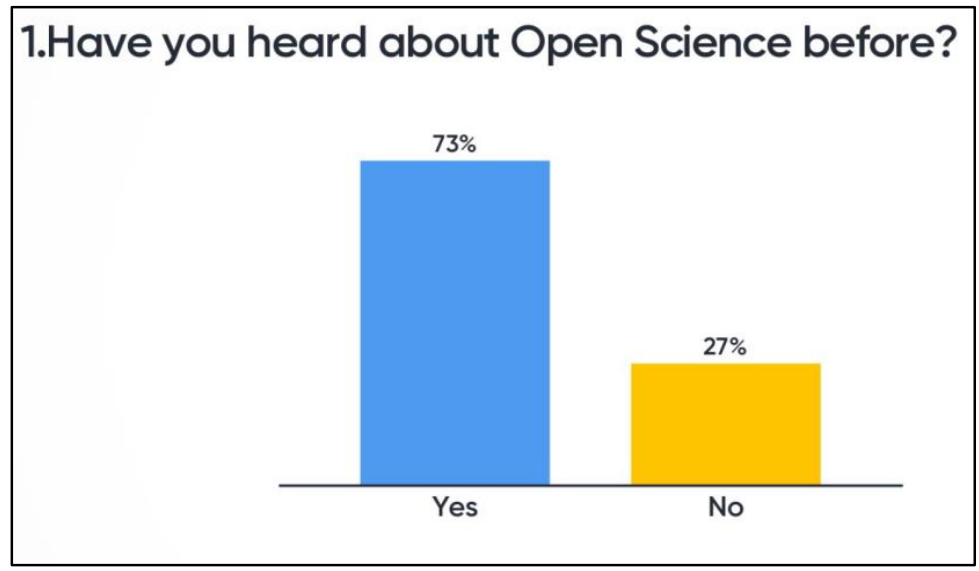

Source: BE OPEN Project - $1^{\text {st }}$ workshop (2019)

Fig. 3 Benefits of OS in transport stakeholders

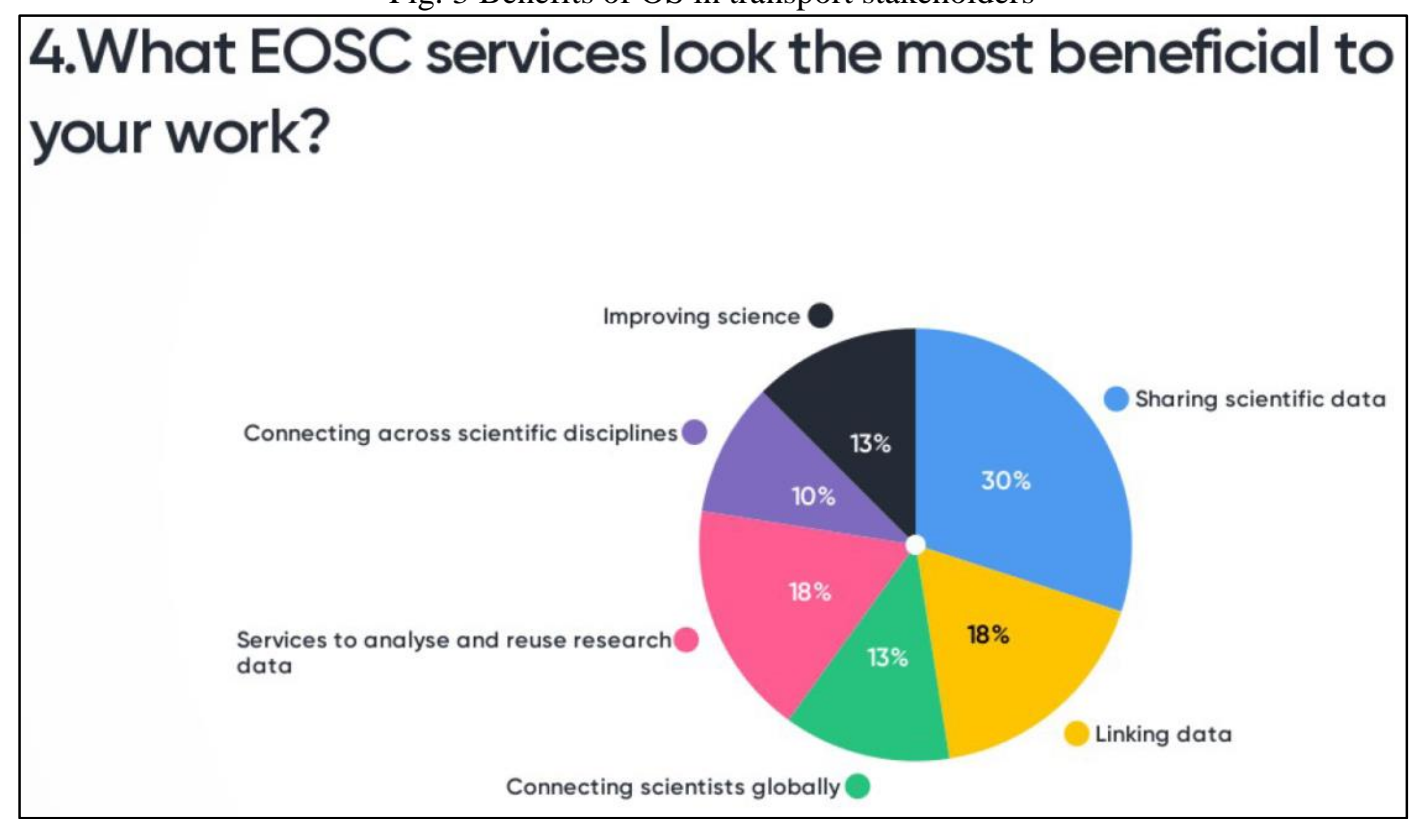

Source: BE OPEN Project - 1st workshop (2019)

In the context of the $1^{\text {st }}$ workshop of the BE OPEN project (BE OPEN Project - 1st workshop, 2019), transport stakeholders have been engaged into the discussion of OS in Transport and more particularly around the terminology for OS understanding and around the needs of the different modes of transport. In particular, the majority of transport stakeholders are aware of OS (Fig. 2) and they can understand the potential benefits that it offers such as to improve their science, to enable sharing of scientific data, to allow link of data, to connect 
scientists at a global level, to provide services that analyze and reuse research data as well as to enhance connectivity across scientific disciplines (Fig. 3). However, they admitted that they are not really interested in promoting, contributing and adopting an "open approach" in the transport sector (Fig. 4). This indicates that a topdown approach is recommended for implementing OS in transport research. There are different OS initiatives and they are difficult to be compared. Hence, there is a need for standardization so as transport research stakeholders to follow a coherent policy that defines the dimensions of OS in transport research and assigns various goals to them. A set of rules and guidelines should be developed to outline how research data will be handled, describing what data will be collected, processed or generated and following what methodology, whether and how these data will be shared and/or made open, and how they will be curated and preserved.

Fig. 4 Interest of Transport Stakeholders in OS

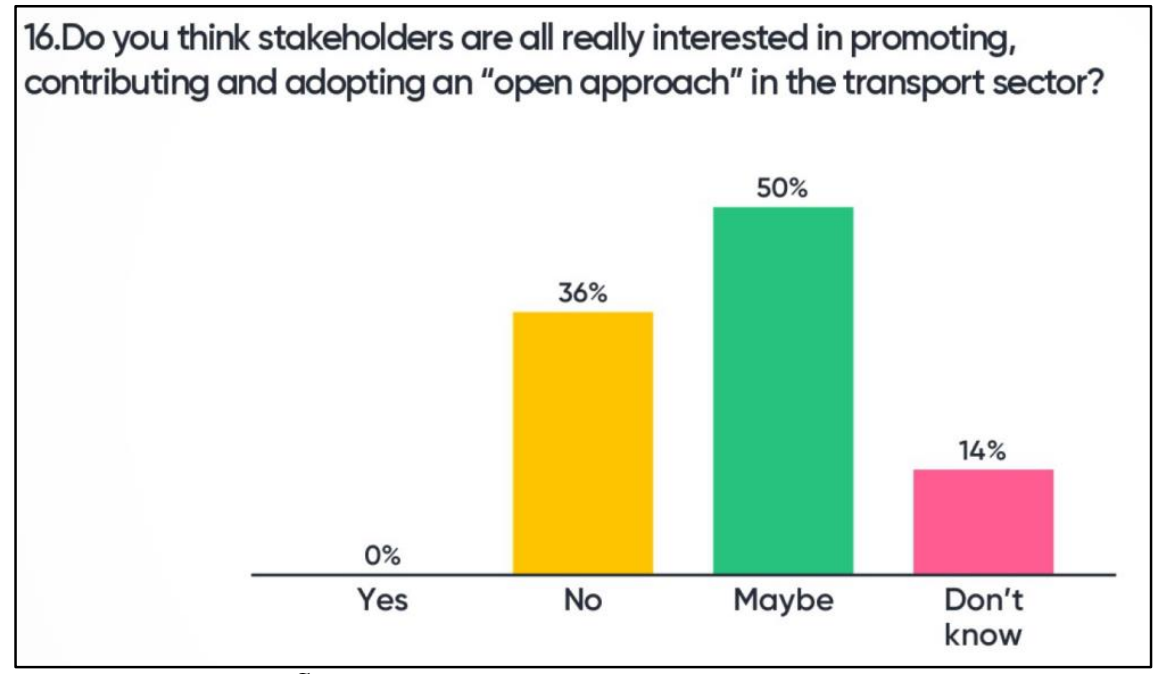

Source: BE OPEN Project - $1^{\text {st }}$ workshop (2019)

\section{Conclusions}

The proposed relations table of the different OS and open access initiatives in transport research (Table 1) provides a starting grid that has to be taken into consideration from key actors for promoting OS in transportation sector. The main goal is to present the latest actions made by EC and private organizations and how each one of them tackles different limitations and challenges of today needs for clarity and transparent use of knowledge and data. These suggestions should make science more efficient, better reproducible and more responsive to societal and economic expectations, as it follows the agenda of EC strategy and promotes collaboration schemes among industry, research community and citizens in order to speed up the path from research to innovation. Besides the aforementioned observations, consideration should be also given towards a systematic review of current European technology platforms and transport research competence areas of key stakeholders as they can give an overall guidance towards a more comprehensive evaluation of current and on-going OS initiatives.

Overall, this study provides an analysis and evaluation of exploited Open Science initiatives and open access platforms in Europe and recommends a more precise definition of OS in transport to specify its overarching goals. A top-down approach is also recommended for implementing OS in transport research to provide transparent and understandable processes and goals to the transport research stakeholders.

\section{Acknowledgements}

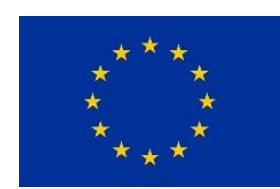

This project has received funding from the European Union's Horizon 2020 research and innovation programme under grant agreement No 824323. The results in this paper reflect only the authors' view. Neither the Innovation and Network Executive Agency nor the European Commission is responsible for any use that may be made of the information contained therein. 


\section{References}

Anagnostopoulou, A., Boile, M., 2018. Challenges and opportunities of open science in transport research. Proceedings of Quaesti Virtual Multidisciplinary Conference 6, 1. Access at: https://www.fosteropenscience.eu/foster-taxonomy/open-science

BE OPEN Project (a), 2019. European forum and oBsErvatory for OPEN science in transport report. Access at: https://cordis.europa.eu/project/rcn/219760/factsheet/en

BE OPEN Project (b), 2019. D2.1 Open access publications and the performance of the European transport research. Access at: https://beopen-project.eu/storage/files/beopen-d21-open-access-publications-and-the-performance-of-the-european-transportresearch.pdf

BE OPEN Project (c), 2019. D2.2 Open/FAIR data, software and infrastructure in European transport research. Access at: https://beopenproject.eu/storage/files/beopen-d22-open-fair-data-software-and-infrastructure-ineuropean-transport-research.pdf

Community Research and Development Information Service (CORDIS), 2019. https://cordis.europa.eu/

BE OPEN Project $-1^{\text {st }}$ workshop, 2019. Open Science in Transport: Challenges and Way Forward. Accessed at: https://beopenproject.eu/events/open-science-session

European Open Science Cloud Project, 2018. Available at: https://ec.europa.eu/research/openscience/pdf/eosc_strategic implem entation roadmap large.pdf\#view=fit\&pagemode=none

Expert Group on Open Science - $\quad$ Transport Research Cloud, $2017 . \quad$ Available at: http://ec.europa.eu/transparency/regexpert/index.cfm?do=groupDetail.gr oupDetail\&groupID=3570\&news=1

FOSTER, 2018. FOSTER portal project. Access at: https://www.fosteropenscience.eu/foster-taxonomy/open-science

GIPO, 2015. Available at: http://observatory.giponet.org/

IETF, 2014. Available at: https://www.ietf.org/about/who/

OpenAIRE portal services, 2016. Available at: https://www.openaire.eu/public-documents/deliverables-all/deliverables

OSCAR, 2019. Open ScienCe Aeronautic \& Air Transport Research project. Available at: https://cordis.europa.eu/project/rcn/219256/factsheet/en

Research Data Alliance (RDA), 2019. Available at: https://www.rd-alliance.org/about-rda

Transport Research Cloud, 2017. Expert Group on Open Science - Transport Research Cloud. Available at: http://ec.europa.eu/transparency/regexpert/index.cfm?do=groupDetail.groupDetail\&groupID=3570\&news=1

Transport Research and Innovation Monitoring and Information System (TRIMIS), 2019. Available at: https://trimis.ec.europa.eu/

Tsakalidis, A., Gkoumas, K., Pekar, F.,Grosso, M, Haq, G.,Marelli, L., 2018. “EU Transport Research \& Innovation Status Assessment Report 2017", Publications Office of the European Union. 